\title{
THRESHOLDING BASED TECHNIQUE FOR RETINAL BLOOD VESSEL EXTRACTION
}

\author{
N. D. Salih \\ Faculty of Computing and Informatics, Multimedia University, \\ Cyberjaya, Selangor, Malaysia \\ E-mail: nbhan@mmu.edu.my \\ Wan Noorshahida Mohd Isa \\ Faculty of Computing and Informatics, Multimedia University, \\ Cyberjaya, Selangor, Malaysia \\ E-mail:wan.norshahida@mmu.edu.my \\ Marwan D. Saleh \\ Electronics and Computer Science University of Southampton Malaysia \\ Johor, Malaysia \\ E-mail: M.D-Saleh@soton.ac.uk
}

\begin{abstract}
The challenging problem of designing techniques that assist computer-aided medical diagnosis has developed as a result of the rapid expansion of computing and information technologies. Due to the obvious growing number of diabetic retinopathy (DR) patients, automated procedures for rapid screening are in greater demand. The accurate diagnosis of DR depends upon detecting and analyzing some features of human retina (i.e. blood vessels, fovea, optic disc, etc.) as well as several types of spot lesions (i.e. exudates, drusen, microaneurysms, hemorrhage, etc). This study focus on blood vessel segmentation in the human retina, which is an essential milestone in retinal image analyzation. To accomplish more efficient and optimum segmentation, the proposed approach employs a number of image processing techniques including contrast enhancement, normalisation, and thresholding. Experiments show that the presented technique produces high-accuracy segmentation and the algorithm is ideally suited for real-time screening applications and large data retrieval.
\end{abstract}

Keyword: Diabetic Retinopathy; Vessel Segmentation; Automatic Thresholding.

\section{Introduction}

Medical imaging enables scientists and clinicians to get a better understanding of potentially life-saving information with less intrusive procedures, and it may reduce the strain of expert labelling dramatically. There is growing awareness and concern about the large and escalating burden of diabetes. The World Health Organization (WHO) reported that there is over than 420 million people worldwide with diabetes. This number is predicted to reach 570 million by 2030 and further to 700 million by 2045. It has also been reported that one in two adults with diabetes are unaware of their condition and are at great risk of debilitating complications that can be prevented through diagnosis [WHO, (2021)].

Diabetic retinopathy (DR) is vision threatening in which damage occurs to the retina due to diabetes mellitus. This disease was ranked as the fifth leading cause of partial or even complete blindness among adults aged 20-74 years [Lee et al. (2015)].

DR is a silent illness that may not be realized by the patient until the retinal abnormalities have progressed to the point where therapy is difficult or impossible. Although diabetes may not be avoided, its vision-threatening consequences can be controlled if the DR is diagnosed and treated early. Early and thorough retinal screening, followed by laser therapy, can greatly minimize the risk of losing visual capability. Regrettably, because visual impairment is a late symptom of severe diabetic retinopathy, many diabetics remain undetected even as their condition progresses and causes serious retinal damage according to [Salih et al. (2018)].

DR falls into two main types; First type is the background retinopathy, consisting of microaneurysms/ haemorrhage (black spots), hard exudate (white spots), retinal edema, and sometimes microinfarcts of the retina 
(cotton wool spots). The second type of DR is the proliferative retinopathy where changes to blood vessel morphology occur, including venous beading (thickening) and the development of new, fine, tortuous vessels [Hann \& Revie, (2015)].

A variety of automated blood vessel segmentation methods have been reported in the literature. Pattern recognition-based, model-based, vessel tracking-based, neural network-based, and artificial intelligence-based approaches are some of the many types of methods available [Kirbas \& Quek, (2004)].

Multi-scale-based, skeleton-based, Region growing, Matching filters, Morphological-based and more subcategories fall under the pattern recognition type. Multi-scale methods segment images at various resolutions. The improved processing speed is the primary benefit of this approach. Low-resolution images are used to obtain the major blood vessels, whereas high-resolution images are used to obtain the tiny blood vessels [Bendaoudi et al. (2016)] and [Dharmawan and $\mathrm{Ng}$ (2018)]. Blood vessel centerlines are obtained using skeleton-based approaches. By linking these centerlines, the vessel tree is produced. To extract the centerline structure, many techniques are employed such as thresholding followed by object connectivity and graph description-based extraction [Kristína et al. (2017)] and [Gao et al. (2020)]. Region growth begins with a seed point in region growing-based pattern recognition algorithms, and the image is subsequently segmented depending on some predetermined criterion [Bhuiyan et al. (2017)]. Ridge-based methods use grayscale images as a height map, with intensity ridges representing the tubular objects' central skeleton. As a result, a two-dimensional image may be perceived as a three-dimensional surface [Staal et al. (2004)]. The geometry-based methods interpret pictures as hypersurfaces and extracts image characteristics using the surface's curvature and crest lines. The crest surface's points correspond to the vessel's centre lines. techniques devised in the field of Differential Geometry are used in these methods [Klein et al. (2018)]. Matching filters techniques of pattern recognition, the retinal image is convolved with several matching filters that hat facilitate the detection of blood vessel segments. [Chaudhuri et al. (1989)], who employed 12 templates to represent the vessel profile, were one of the first to apply filteringbased vessel segmentation techniques [Elson et al. (2017)] [Kadry et al. (2021)]. Morphology is related to the study of object shape. Morphology-based methods facilitates the segmentation and searching for objects of interest by Filing holes and removing extraneous segments. To govern the key Morphological operators, such as dilation and erosion, specific structural components are added to images. These operations these operators are usually applied to binary images, however there are several exceptions for gray-level images [Chouchene et al. (2018)].

In contrast to pattern recognition methods, vessel-tracking methods use local operators to segment the predicted blood vessel rather than global operators. These approaches attempt to segment the vessels by following the vessels' centre line. Different vessel estimating profiles, including Bayesian probabilistic, Gaussian and Generic parametric can be used to trace the vessel. [Vincent et al. (2019)].In skeleton-based approaches, the vasculature is extracted using explicit vessel models. Snake algorithm is the most fundamental blood vessel extraction algorithms. Four sub-categories within this category, they are: Deformable model-based techniques, parametric model-based techniques, template matching techniques, and generalised cylinders techniques [Mukaida et al. (2020)].

As classification algorithms, neural network-based approaches are employed. The network weights are determined by training the system on a database of images with blood vessel outlines, and the trained system is then used to segment the target retinal image [Fu et al. (2020)]. Artificial intelligence-based methods undertake the segmentation process and then outline the vessel structures using prior knowledge of a model blood vessel structure. [Aslan et al. (2018)] and [Gojić et al. (2020)]. Readers interested in blood vessel extraction and analysis methods are directed to a variety of surveys, including [Khan et al. (2019)] and [Memari et al. (2019)].

This study focus on blood vessel segmentation in the human retina, which is an essential milestone in the analysis of retinal images. Low contrast in fundus images can be caused by a variety of circumstances, the proposed algorithm employs the Contrast Limited Adaptive Histogram Equalization (CLAHE) technique to conduct contrast enhancement. An Automatic Thresholding $(A T)$ technique, called Isodata, is also employed in the proposed algorithm to facilitate blood vessels segmentation. The algorithm is particularly well suited for realtime screening applications and large data retrieval, as it employs simple pre-processing techniques. The rest of this paper is organized as follows: Section 2 begins with a brief overview of the method, followed by a discussion of the results in Section 3, and lastly, a conclusion in Section 4.

\section{Methodology}

In this paper, we have devised an algorithm for segmenting retinal blood vessels automatically. Based on the proposed Algorithm a color fundus photography of human retina is fed into the system that consequently produces a binary image comprising just blood vessels as an output. The algorithm consists of four main steps: 1) Convert RGB fundus image into grayscale/green, 2) contrast enhancement, 3) background normalization, 4) thresholding and post filtration. The algorithm allows producing either the gray-scale or green-channel blood 
vessels component of the RGB fundus image. The main phases of the presented algorithm are depicted visually in Fig. 1.

\subsection{RGB to Grayscale/Green channel Conversion}

As a first step, the proposed approach transforms the colour fundus image into grayscale or green-channel image first, which facilitates blood vessel segmentation process and reduces computational cost. A comparison of the results based on gray and green channel components is given in Section 3. Converting RGB fundus images to grayscale eliminates the hue and saturation information from the three channels while preserving their luminance.

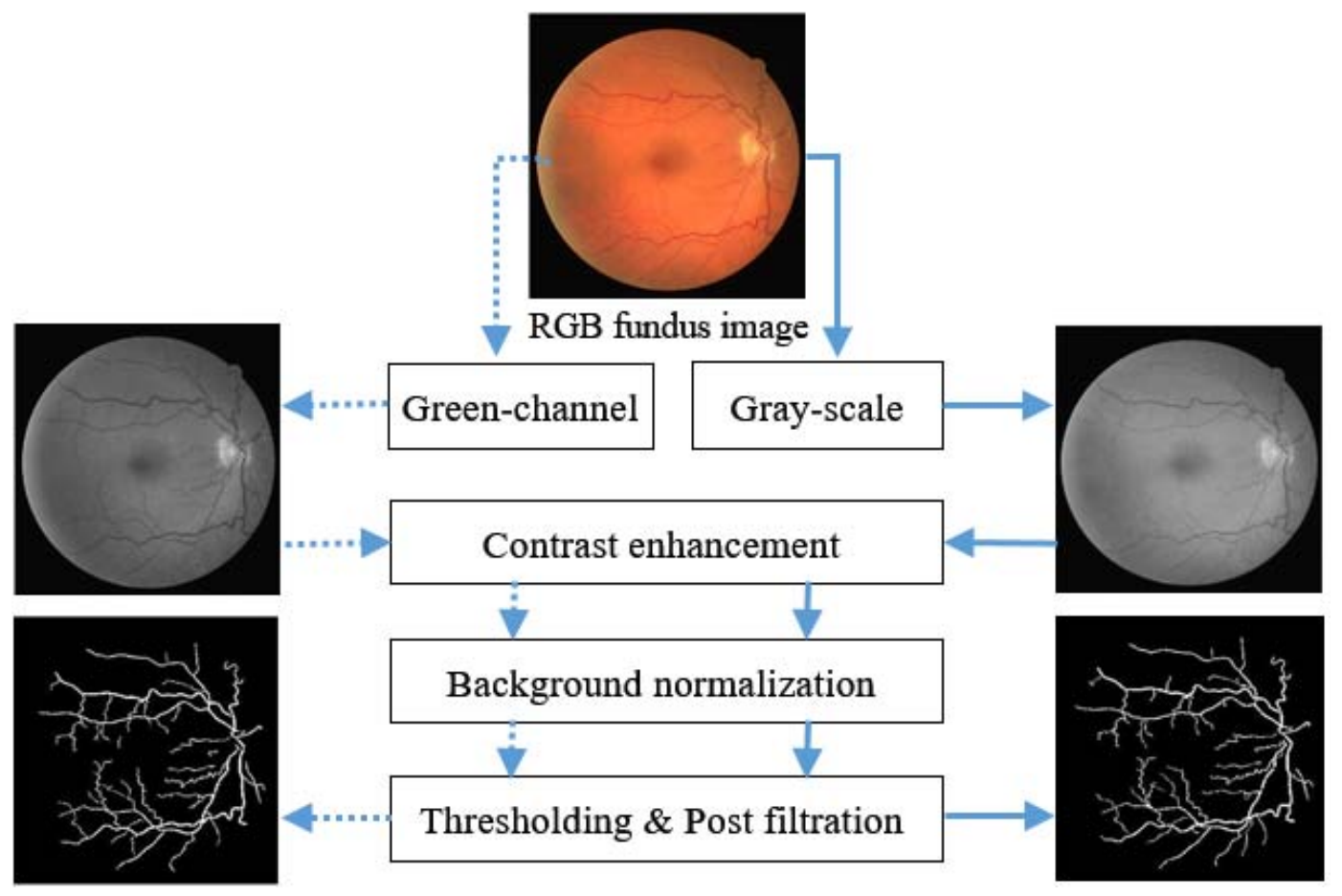

Fig. 1. Phases of the proposed algorithm

In addition, the green channel exhibits the largest local contrast between the blood vessels and the retinal background when compared to the red and blue channels [Jorge et L. (2003)]. The gray-scale image and greenchannel image, extracted from an RGB fundus image can be seen in Fig.2.

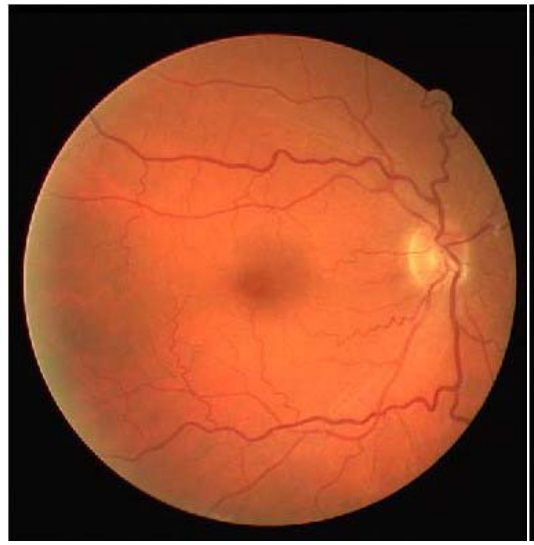

(a)

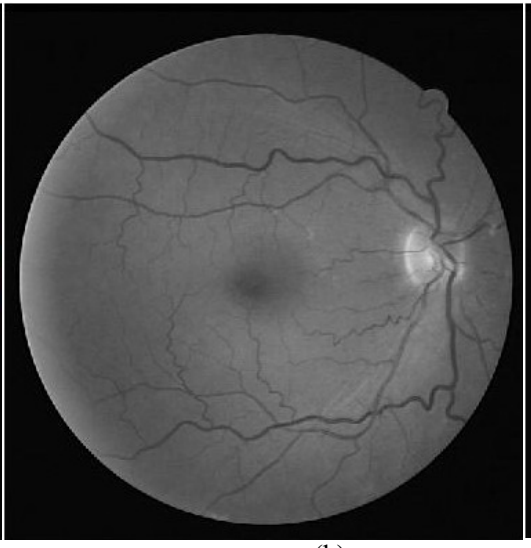

(b)

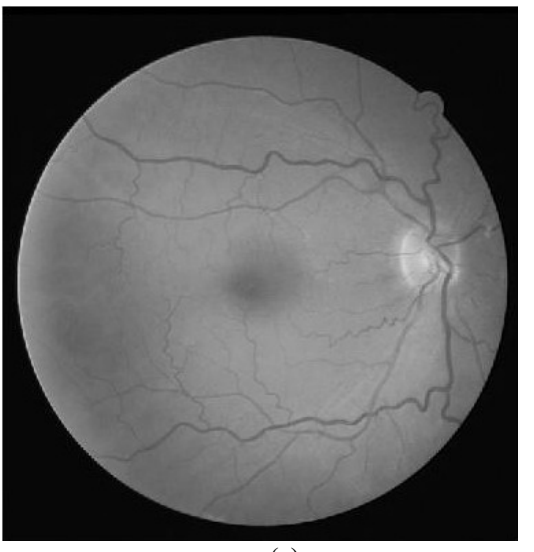

(c)

\subsection{Contrast Enhancement}

Low contrast in fundus images can be caused by a variety of circumstances, including hardware/software issues, as well as fluctuations in the intensity caused by the surrounding lighting, resulting in poor segmentation. Contrast stretching is an image enhancement technique that aimed at increasing image contrast by broadening the image's range of intensity values to span the image's full dynafmic range and, as a result, improve the contrast of the fundus image before further processing [Jain, (1989)] and [Pizer et al. (1990)]. 
There has been a wide range of proposed contrast enhancement techniques, which may be categorized into spatial domain and transform domain techniques, for example: Median filters, Decorrelation-stretching transform, Unsharp mask, 2D empirical mode decomposition, Histogram equalization (HE), Adaptive Histogram equalization (AHE), and a variation of AHE called (Contrast-Limited AHE) or (CLAHE). Among other techniques Fourier-based and wavelet-based are transform domain examples [Zhou et al. (2019)], [Wang, (2017)], and [Yang et al. (2010)].

Rather than working on the entire image, CLAHE works on small regions of the image called tiles. The image is initially divided into tiles, and after that, histogram equalization is applied to each one for contrast enhancement. Finally, using bilinear interpolation, the neighbouring tiles are merged together to generate a more contrasted output image. Many studies have employed CLAHE as a processing technique to boost contrast in fundus images, and it has proven to be quite successful [Ooi et al. (2021)], [Adapa et al. (2020)] and [Papadopoulos et al. (2008)]. The proposed technique employs the CLAHE technique to conduct contrast stretching. CLAHE contrast stretching was applied to both gray-scale and green-channel images as shown in Fig. 3.

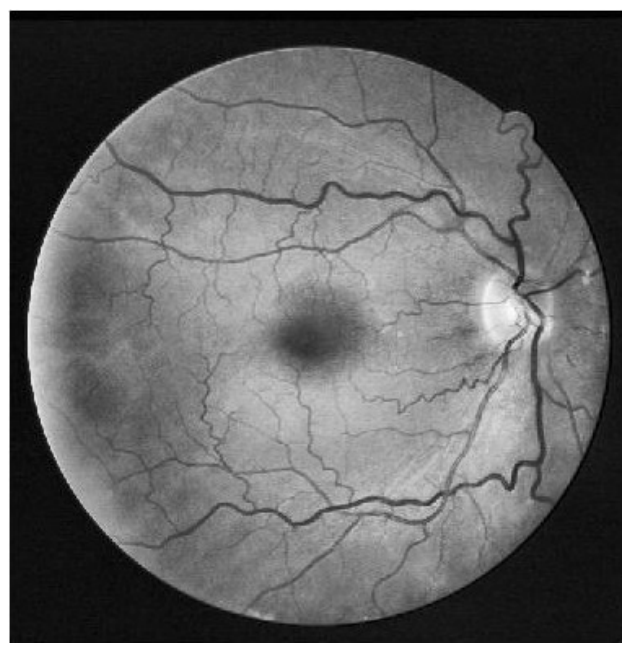

(a)

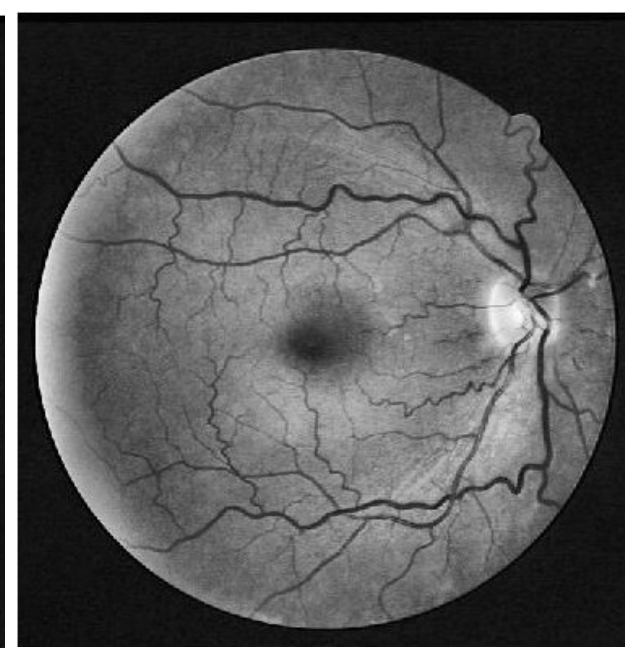

(b)

Fig. 3. CLAHE's contrast stretching on (a) Gray-scale (b) Green-channel.

\subsection{Background Normalization}

Background Background normalization is basically used for enhancing images adaptively by eliminating featureless, non-uniform background illumination, or in other words, re-distributing the intensity levels of the background within a converging range [Salih et al. (2017)]. Consequently, this process would lead to easier segmentation of blood vessels. Background normalization is performed as follows:

1) Filtering the gray/green image $g$ using an average filter to produce a new image $\hat{g}$.

2) Subtract the image $g$ from the average-filtered image $\hat{g}$ to obtain the background normalized image $h$.

The above steps are implemented as in Eqs.(1) and (2) (Costa \& Cesar (2001), (Kwan, 2003) and Gonzalez \& Woods (2002)).

$$
\begin{gathered}
\hat{g}(x, y)=\frac{1}{M \times N} \sum_{i=1}^{M \times N} g_{i}(x, y) \\
h=\hat{g}-g
\end{gathered}
$$

where $M \mathrm{x} N$ represents the window size.

\subsection{Thresholding and Post Filtration}

In digital image processing, thresholding is a process of segmenting an image contents into a foreground (black) and background (white), replacing individual pixels with black pixels when the intensity of the pixels falls below a pre-set value $T$ (threshold value) or with white pixels when the intensity exceeds that value. From the obtained grayscale image $\mathrm{h}$, Thresholding generates a binary image in which each pixel's value is either 0 (represents background) or 1 (represents vessel). Two thresholding techniques are employed in our algorithm for producing the binary image $f(x, y)$. The first is based on a fixed threshold value $T$, whereas the second is based on an automatic thresholding. Generally, for each normalized background image $h(x, y)$ 


$$
f(x, y)= \begin{cases}1 \text { if } & h(x, y) \geq T \\ 0 & \text { otherwise }\end{cases}
$$

where $0 \leqslant h(x, y) \leqslant 255$.

In the fixed threshold $(F T)$ technique, $T \approx 0$ since in the image $h(x, y)$, the intensity values of the background pixels approximate to 0 and most of the other pixels with intensity values greater than 0 belong to the blood vessels region. An automatic thresholding $(A T)$ technique, called Isodata [Ridler \& Calvard (1978)] is also used in the proposed algorithm. This technique starts by partitioning the image histogram into two partitions, $P_{1}$ and $P_{2}$ based on an initial threshold value $T_{0}$ (usually, $T_{0}$ the average intensity of the image). Subsequently, the mean intensity values $\mu_{1}$ and $\mu_{2}$ are calculated for $P_{1}$ and $P_{2}$ respectively, and uses the average of $\mu_{1}$ and $\mu_{2}$ as a new threshold value $T_{k}$, as shown in Eq.4. The new threshold value is updated iteratively by the new average intensity values until the convergence of $T_{k}$ and $T_{k-1}$ is achieved (i.e. $T_{k} \approx T_{k-1}$ )

$$
T_{k}=\frac{\mu_{1}+\mu_{2}}{2}
$$

Figs.4 (a) and (c) show the resulting binary images for gray/green channels obtained using the automatic thresholding technique.

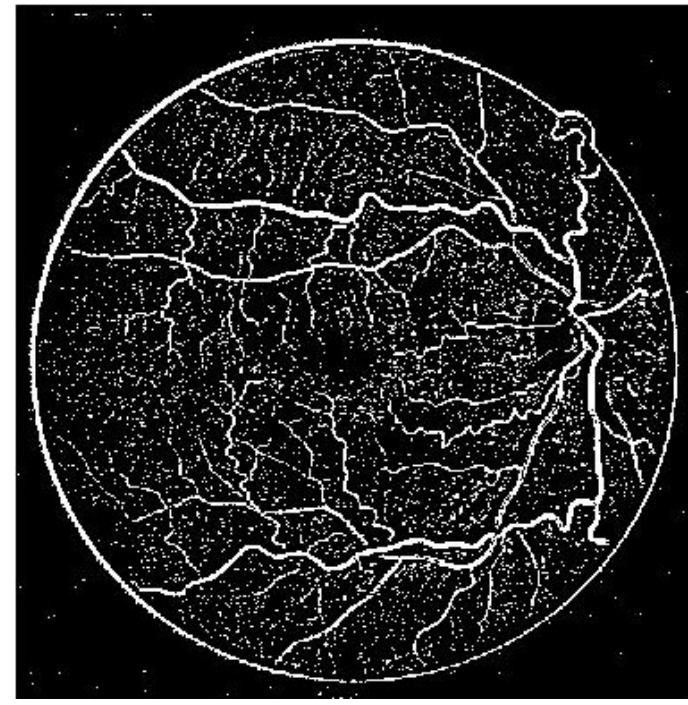

(a)

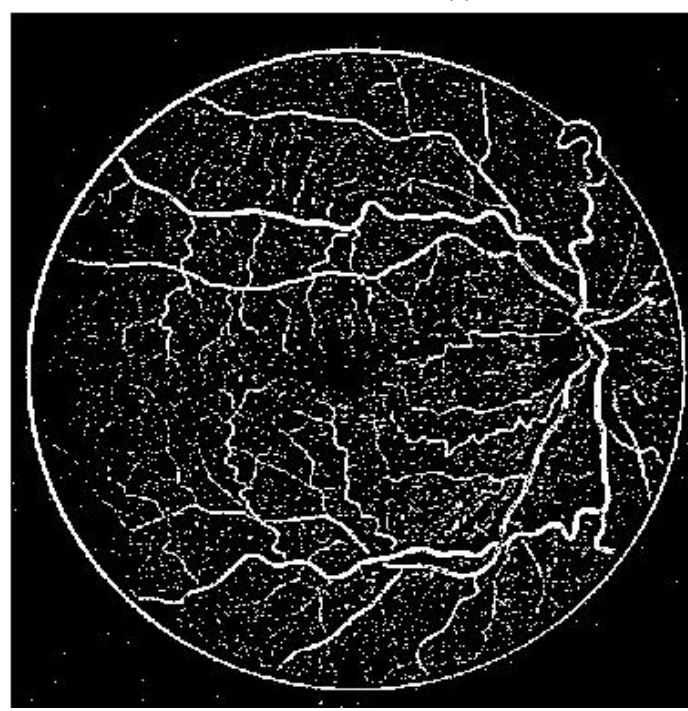

(c)

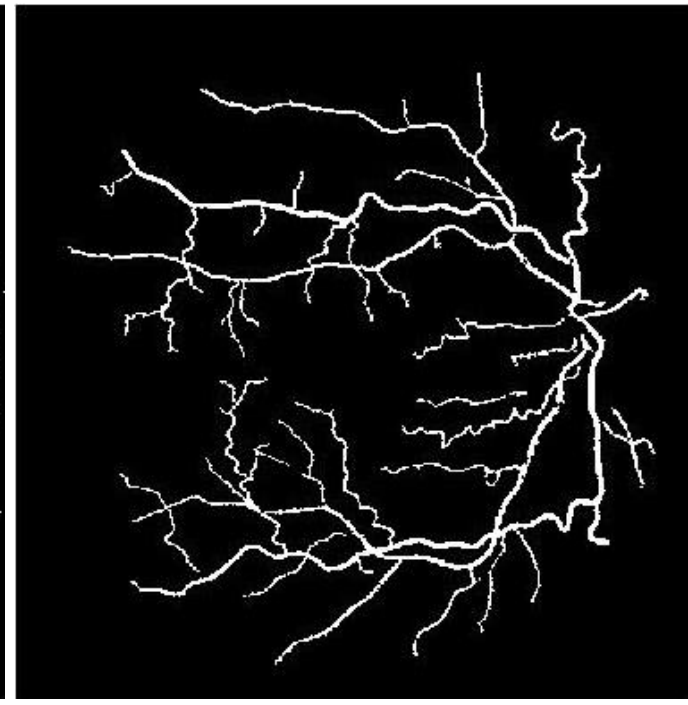

(b)

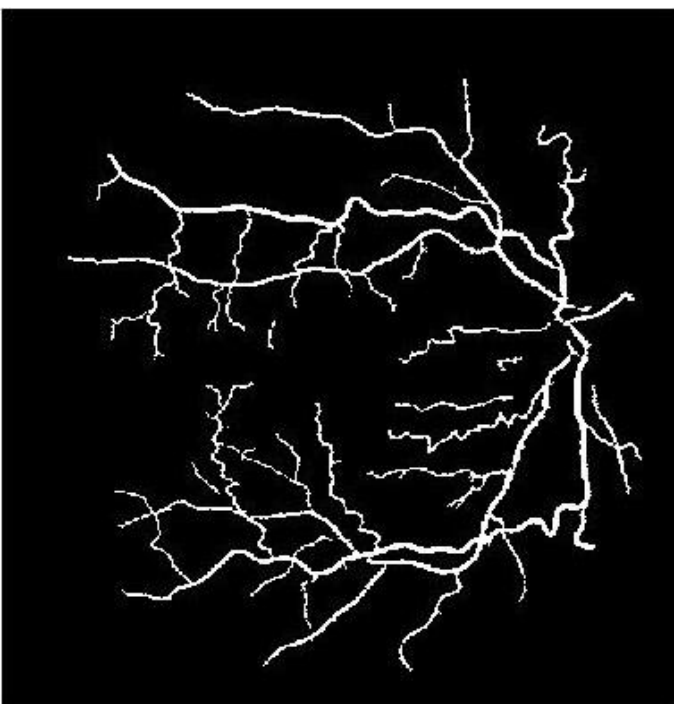

(d)

Fig. 4. (a) binary gray-scale image + auto thresholding (b) binary gray-scale filtration (c) binary green-channel image + auto thresholding (d) binary green-channel filtration. 
In most cases, applying a thresholding technique produces inadequate binary images due to some undesired small objects or pixels that appear as noise. This noise constitutes obstacles to posterior processing of the images; therefore, post-processing and Filtration techniques for suppressing unpredictable noise should be carried out in order to achieve a better image quality. For this purpose, an operation known as morphological opening is engaged. This operation refines the binary image by removing small and isolated objects while preserving the shape and size of larger objects [Papadopoulos et al. (2008)]. The results of applying post filtration and noise reduction are shown in Fig. 4 (b) and (d).

\section{Results and Discussion}

In our experiment, the training set of retinal fundus images of the DRIVE dataset [Staal et al. (2004)] are tested to evaluate the proposed algorithm. DRIVE database is commonly in use for vessel segmentation research. The images for the DRIVE dataset were collected from a diabetic retinopathy screening system in the Netherlands for Diabetic individuals aged 25 to 90 years. The training set contains 20 retinal images of size $565 \times 584$ pixels captured in a 45-degree field of view by a Canon CR5 camera. For each of the training images, a reference manual image is available for the performance comparison. The performance is validated using two common criteria: The true positive fraction $(T P F)$ and the false positive fraction $(F P F)$. TPF denotes the percentage of pixels properly identified as blood vessel pixels. while the percentage of non-vessel pixel identified wrongly as vessel pixels is denoted by FPF. TPF and FPF are given by Eqs. (5), (6) respectively [Marshall \& Bangert (1995)].

$$
\begin{aligned}
& T P F=\frac{T P}{T P+F N} \\
& F P F=\frac{F P}{F P+T N}
\end{aligned}
$$

Where the TP value represent true positive, the $F N$ value represent false negative, the $F P$ value represent false positive, and finally TN represent true negative values. Figs. 5 and 6 show the $T P F$ and $F P F$ values obtained using the proposed algorithm based on gray and green channels respectively. The figures show the results obtained with fixed thresholding $(F T)$ as well as automatic thresholding $(A T)$.

Table 1 shows the average $T P F$ and $F P F$ values after applying the algorithm for both versions of the images gray-scale and green-channel. The experimental results shown in Table 1 demonstrate that the algorithm yields a high accuracy in terms of TPF and FPF measures.

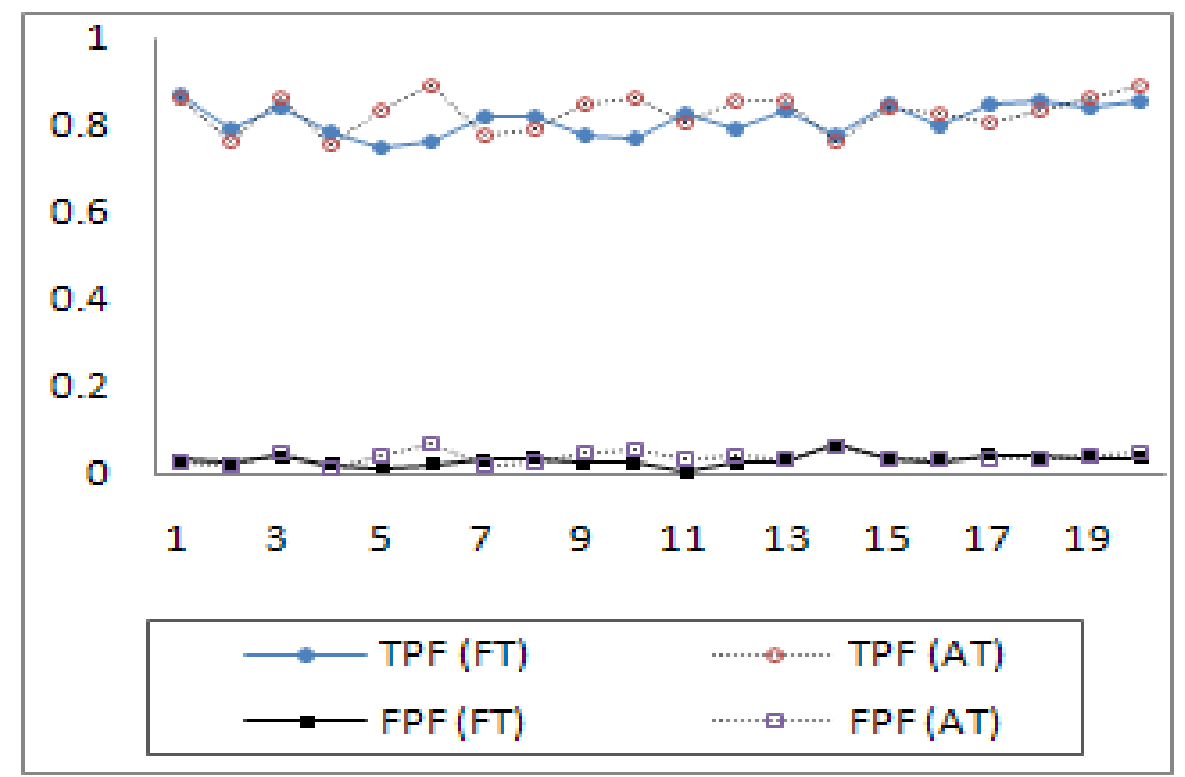

Fig. 5. TPF and FPF measures for gray-scale images (DRIVE database) 


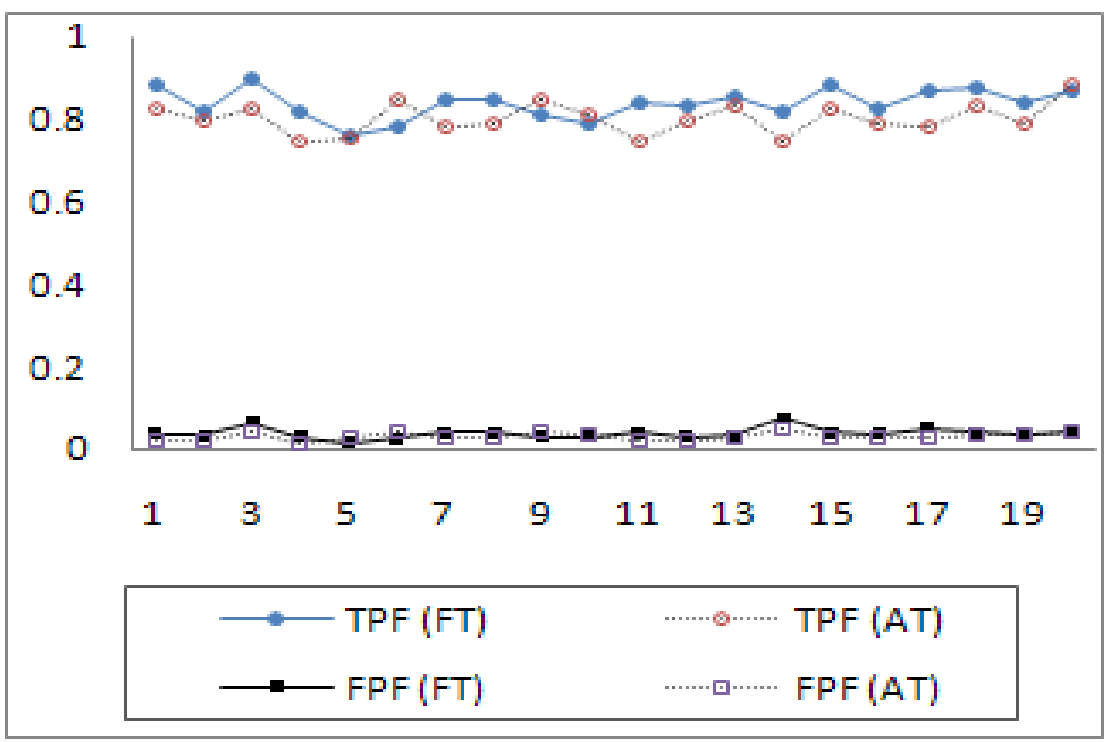

Fig. 6. $T P F$ and $F P F$ measures for Green-channel images (DRIVE database)

Table 1. Average TPF and FPF values obtained

\begin{tabular}{lcc}
\hline Proposed algorithm & TPF & FPF \\
\hline Gray-scale (Fixed $T$ ) & 0.8117 & 0.0291 \\
Gray-scale (Auto. T) & 0.8288 & 0.0363 \\
Green-channel (Fixed $T$ ) & 0.8376 & 0.0383 \\
Green-channel (Auto. T) & 0.8023 & 0.0299 \\
\hline
\end{tabular}

Based on average accuracy measure (Acc), Eq. (7), we compared the performance of our method to those of other methods

$$
A c c=\frac{(T N+T P)}{(F P+F N+T P+T N)}
$$

Table 2 shows the compression results of average accuracy obtained using the proposed algorithm based on gray-scale and green-channel images to those obtained using other well-known algorithms on the Drive database. In terms of average accuracy measure, the proposed method using G-channel clearly surpasses other methods. However, the result of grayscale-based is marginally inferior to those obtained with Dash \& Bhoi (2018), Thangaraj et al. (2018) and Vaidya et al. (2015). Fig. (7) and (8) show some random examples of original and segmented images produced with the proposed algorithm based on G-channel and grayscale respectively.

Table 2. Comparison of performance results on drive database with other methods.

\begin{tabular}{lc}
\hline Method & $\begin{array}{c}\text { Accuracy, } \\
\text { (Acc) \% }\end{array}$ \\
\hline [Adapa et al. (2020)] & 94.50 \\
[Dash \& Bhoi (2018)] & 95.71 \\
[Strisciuglio et al. (2015)] & 94.42 \\
Thangaraj et al. (2018) & 96.06 \\
[Roychowdhury et al. (2015a)] & 94.42 \\
[Roychowdhury et al. (2015b)] & 95.19 \\
[Vaidya et al. (2015)] & 95.60 \\
[Imani et al. (2015)] & 95.23 \\
[Melinscak et al. (2015)] & 94.66 \\
[Fu et al. (2016)] & 95.23 \\
Presented alg. (grayscale) & 95.54 \\
Presented alg. (G-channel) & 96.30 \\
\hline
\end{tabular}



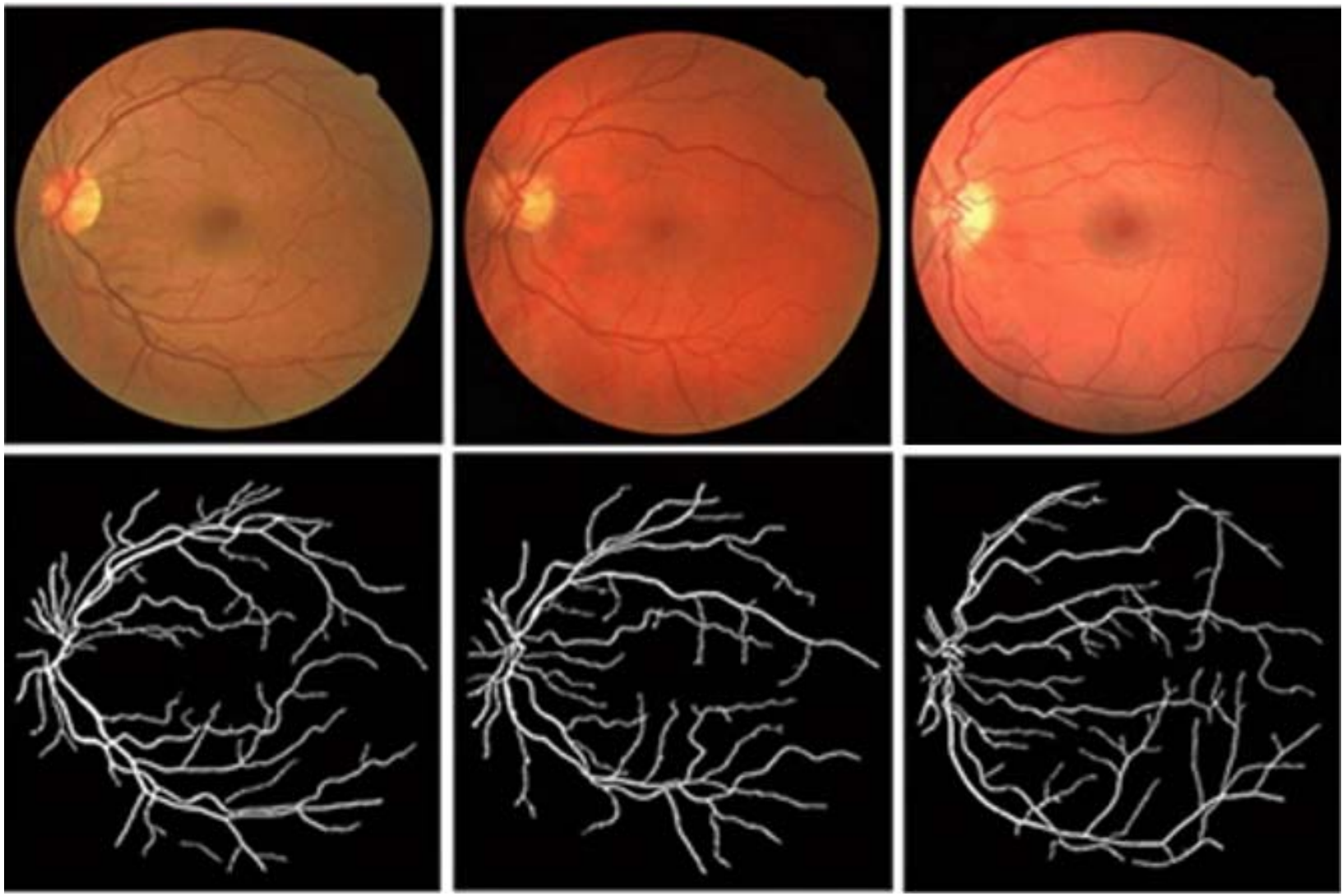

Fig. 7. Random examples of original and segmented images produced with the proposed algorithm based on G-channel.
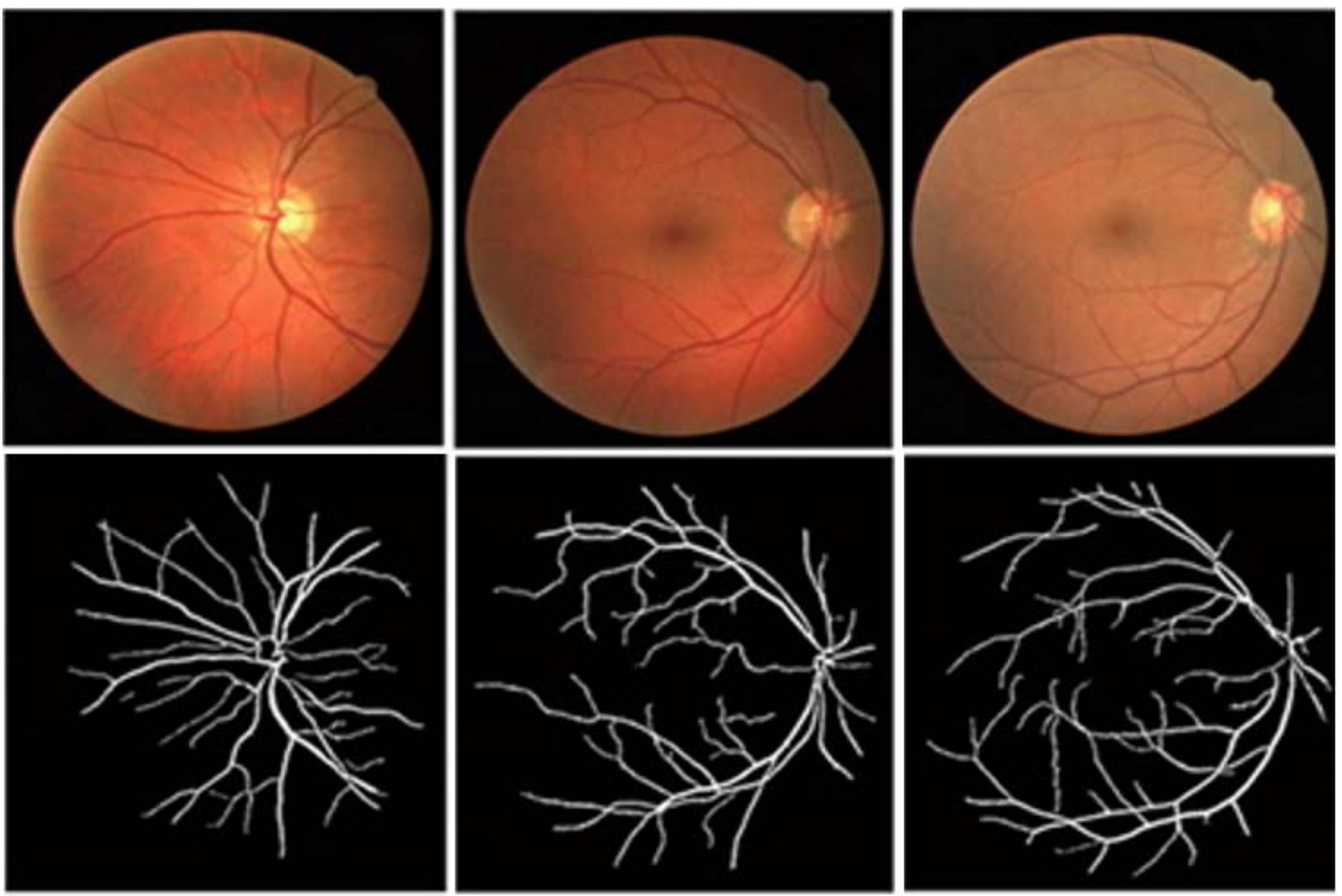

Fig. 8. Random examples of original and segmented images produced with the proposed algorithm based on grayscale images. 
We attribute these remarkable results to the use of a superior image enhancement, thresholding and post filtration techniques that provided an accurate response to the majority of thinner blood vessels segmentation. On the other hand, the employment of uncomplicated pre-processing techniques can significantly reduce the processing time of segmentation compared to techniques that require extensive computation, including, convolutional neural networks and region growing technique. As a result, this algorithm is ideally suited for realtime screening applications and large data retrieval. Using MATLAB R2015a with a $2.80 \mathrm{GHz}$ processor and $4 \mathrm{~GB}$ of RAM, in most cases the average time taken for vessels segmentation approximately 0.25 second per image.

\section{Conclusion}

This article presents a simple, fast, and powerful automatic segmentation algorithm, with this algorithm, a few image-processing techniques for contrast enhancement, normalization, and thresholding, are used. The proposed algorithm may be utilized with either grayscale or green-channel fundus images. Automatic and preset thresholding approaches were used for binarization. To assess the proposed algorithm, retinal images from DRIVE database have been used. The experimental results demonstrate that the proposed algorithm yields high accuracy in terms of TPF and FPF measures. In terms of average accuracy measure, the proposed algorithm outperformed well-known algorithms. The algorithm's simplicity makes it an ideal candidate for real-time screening applications and mass data retrieval.

\section{References}

[1] Adapa D, Joseph Raj AN, Alisetti SN, Zhuang Z, K. G, et al. v. (2020): A supervised blood vessel segmentation technique for digital Fundus images using Zernike Moment based features. PLOS ONE 15(3).

[2] Aslan, M. F.; Ceylan, M. \& A. Durdu (2018): Segmentation of Retinal Blood Vessel Using Gabor Filter and Extreme Learning Machines," 2018 International Conference on Artificial Intelligence and Data Processing (IDAP), pp. 1-5.

[3] Bendaoudi H.; Cheriet F.; and Langlois , J. M. P.,(2016): Memory efficient Multi-Scale Line Detector architecture for retinal blood vessel segmentation. 2016 Conference on Design and Architectures for Signal and Image Processing (DASIP), pp. 59-64.

[4] Bhuiyan, A., Nath, B., \& Chua, J.J. (2017): An adaptive region growing segmentation for blood vessel detection from retinal images. VISAPP. DOI:10.5220/0002054104040409

[5] Chaudhuri, S., Chatterjee, S., Katz, N., Nelson, M., \& Goldbaum, M. (1989): Detection of blood vessels in retinal images using twodimensional matched filters. IEEE Transactions on Medical Imaging, 8(3), 263-269.

[6] Chouchene,A.; Barhoumi,W.; \&Ahmad A. (2021): Retinal blood vessel segmentation in fundus images based on morphological operators within entropy information, Proc. SPIE, 11605, Thirteenth International Conference on Machine Vision.

[7] Christopher E. Hann, James A. Revie. (2009): Screening for Diabetic Retinopathy Using Computer Vision and Physiological Markers. Journal of Diabetes Science and Technology, vol.3(4).

[8] Costa, L. d. F., Cesar Jr., R. M.; (2001): Shape Analysis and Classification: Theory and Practice. CRC Press, ISBN 0-8493-3493-4.

[9] Dash, J., Bhoi, N. (2018); An Unsupervised Approach for Extraction of Blood Vessels from Fundus Images. J Digit Imaging, vol. 31, pp. 857-868.

[10] Dharmawan D. A. and Ng B. P. (2018): Segmenting Retinal Vessels with a Multi-scale Modified Dolph-Chebyshev Type I Function Matched Filter. 4th International Conference on Science and Technology (ICST), , pp. 1-4.

[11] Elson J., Precilla, J., Reshma, P. and Madhavaraja, N. S. (2017): Automated extraction and analysis of retinal blood vessels with Multi Scale Matched Filter," 2017 International Conference on Intelligent Computing, Instrumentation and Control Technologies (ICICICT), pp. 775-779.

[12] Fu H., Xu Y., and Lin S. et al. (2016): Deep vessel: retinal vessel segmentation via deep learning and conditional random field. Proc. Int. Conf. Medical Image Computing and Computer-Assisted Intervention (MICCAI), pp. 132-139.

[13] Fu, Q.; Li. S.; and Wang, X., (2020): MSCNN-AM: A Multi-Scale Convolutional Neural Network with Attention Mechanisms for Retinal Vessel Segmentation," in IEEE Access, vol. 8, pp. 163926-163936.

[14] Gao,j.; Chen, C.; Lin, W. (2020): An Effective Retinal Blood Vessel Segmentation by Using Automatic Random Walks Based on Centerline Extraction, BioMed Research International, vol. 2020,

[15] Gojić G. et al. (2020): Deep Learning Methods for Retinal Blood Vessel Segmentation: Evaluation on Images with Retinopathy of Prematurity, 2020 IEEE 18th International Symposium on Intelligent Systems and Informatics (SISY), pp. 131-136.

[16] Gonzalez, R. C., Woods, R. E.. (2002): Digital Image Processing. 2nd Edition. Prentice hall, ISBN: 0201180758.

[17] Imani E., Javidi M., and Pourreza H.R. (2015): Improvement of retinal blood vessel detection using morphological component analysis. Comput. Methods Programs Biomed, vol. 118, no. 3, pp. 263-279.

[18] Jain, A. K.. (1989): Fundamental of digital image processing", Prentice Hall, ISBN: 0133325764

[19] Jorge J. G. Leandro, Jo ao V. B. Soares, Roberto M. Cesar Jr., Herbert F. Jelinek. (2003): Blood Vessels Segmentation in Non-Mydriatic Images using Wavelets and Statistical Classifiers. in: Proc. of the 16th Brazilian Symposium on Computer Graphics and Image Processing (SIBGRAPI), pp. 262-269.

[20] Kadry S., Rajinikanth V., Damaševičius R. and Taniar D., (2021): Retinal Vessel Segmentation with Slime-Mould-Optimization based Multi-Scale-Matched-Filter," 2021 Seventh International conference on Bio Signals, Images, and Instrumentation (ICBSII), 2021, pp. $1-5$.

[21] Khan, K.B., Khaliq, A.A., Jalil, A. et al. (2019): A review of retinal blood vessels extraction techniques: challenges, taxonomy, and future trends. Pattern Anal Applic 22, 767-802.

[22] Kirbas, C., \& Quek, F. (2004). A review of vessel extraction techniques and algorithms. ACM Computing Surveys (CSUR), 36(2), 81121.

[23] Klein, R., Lee, K. E., Danforth, L., Tsai, M. Y., Gangnon, R. E., Meuer, S. E., Wong, T. Y., Cheung, C. Y., \& Klein, B. (2018). The Relationship of Retinal Vessel Geometric Characteristics to the Incidence and Progression of Diabetic Retinopathy. Ophthalmology, 125(11), 1784-1792. https://doi.org/10.1016/j.ophtha.2018.04.023

[24] Kristína, L.; Hans, F.; Chunliang, W.; Ewert, B.; Örjan, S. (2017): Chapter 12 - Skeleton-based fast, fully automated generation of vessel tree structure for clinical evaluation of blood vessel systems, Editor(s): Punam K.. Academic Press, pp. 345-382

[25] Kwan, H. K. (2003): Fuzzy Filters for Noisy Image Filtering. in: Proc. Int. Sym. on Circuits and Systems (ISCAS), vol. 4, pp 161-164. 
[26] Lee, R., Wong, T.Y. \& Sabanayagam, C. (2015): Epidemiology of diabetic retinopathy, diabetic macular edema and related vision loss. Eye and Vis 2(17).

[27] Marshall, W. J., Bangert, S. K. (1995): Clinical Biochemistry: Metabolic and Clinical Aspects. Elsevier Health Sciences, ISBN 0443043418

[28] Melinscak M., Prentasic P., and Loncaric S. (2015): Retinal vessel segmentation using deep neural networks. Proc. 10th Int. Conf. Computer Vision Theory and Applications (VISAPP 2015). Berlin, Germany, vol. 1, pp. 11-14.

[29] Memari, N. et al. (2019): Retinal Blood Vessel Segmentation by Using Matched Filtering and Fuzzy C-means Clustering with Integrated Level Set Method for Diabetic Retinopathy Assessment. J. Med. Biol. Eng. 39, 713-731.

[30] Mukaida, M. ; Okami, Y.; Suetake N. and Uchino, E. (2020): Contour Line Extraction of Vein with Use of Special Tracking Strategy and Fuzzy Inference for Arteriosclerosis Diagnosis of Retinal Blood Vessel," IECON 2020 The 46th Conf.of the IEEE Industrial Electronics Society, pp. 435- 440.

[31] Ooi,et al. (2021): Interactive Blood Vessel Segmentation from Retinal Fundus Image Based on Canny Edge Detector. Sensors. 21(19).

[32] Papadopoulos, A., Fotiadis, D.I., Costaridou, L. (2008): Improvement of microcalcification cluster detection in mammography utilizing image enhancement techniques. Journal of Computers in Biology and Medicine, 38, pp. $1045-1055$.

[33] Pizer, S. M., Johnston, R. E., Ericksen, J. P. (1990): Contrast-Limited Adaptive Histogram Equalization: Speed and Effectiveness. in: Proc. of the 1st Conf. on Visualization in Biomedical Computing, pp. 337-345.

[34] Pizer, S. M., Johnston, R. E., Ericksen, J. P., Yankaskas, B. C. (1990): Contrast-Limited Adaptive Histogram Equalization: Speed and Effectiveness. in: Proc. of the 1st Conf. on Visualization in Biomedical Computing, pp. 337-345.

[35] Ridler T.W., Calvard, S. (1978): Picture thresholding using an iterative selection method. in: Proc. IEEE Trans. On Systems, Man, Cybernetics, vol. SMC-8, pp. 630-632.

[36] Roychowdhury, S., Koozekanani D. D. and Parhi, K. K.. (2015a): Blood Vessel Segmentation of Fundus Images by Major Vessel Extraction and Subimage Classification. in IEEE Journal of Biomedical and Health Informatics, 19(3), pp. 1118-1128.

[37] Roychowdhury, S., Koozekanani, D. D. and Parhi, K. K., (2015b); Iterative Vessel Segmentation of Fundus Images. in IEEE Transactions on Biomedical Engineering, 62(7), pp. 1738-1749.

[38] Salih, N. D., Saleh, M. D., Eswaran, C., and Abdullah, Junaidi. (2015): Fast Optic Disc Segmentation Using FFT-Based TemplateMatching and Region-Growing Techniques. Computer Methods in Biomechanics and Biomedical Engineering: Imaging \& Visualization, 6(1): 101-112.

[39] Salih, N. D., Saleh, Marwan D., Eswaran, C., and Abdullah, Junaidi. (2017): Grading System for Diabetic Retinopathy Disease. International Journal of Signal Processing Systems, 5(1):34-38.

[40] Saranya, s., \& ellappan, v. (2017): Retinal blood vessels separation - a survey. Indian journal of scientific research, 17 (1): $142-151$.

[41] Staal, J., Abràmoff, M. D., Niemeijer, M., Viergever, M. A., \& Van Ginneken, B. (2004): Ridge-based vessel segmentation in color images of the retina, IEEE Transactions on Medical Imaging, 23(4), 501-509.

[42] Staal, J.J., Abramoff, M.D., Niemeijer, M., Viergever, M.A., Ginneken B. van, (2004): Ridge based vessel segmentation in color images of the retina", IEEE Transactions on Medical Imaging, 23, pp. 501-509. Drive database [Online]. Available: https://drive.grandchallenge.org/

[43] Strisciuglio N, Vento M, Azzopardi G, Petkov N.(2015): Unsupervised delineation of the vessel tree in retinal fundus images. Proceedings of the 5th Eccomas Thematic Conference on Computational Vision and Medical Image Processing, pp.149-155.

[44] Thangaraj S, Periyasamy V, Balaji R.. (2018): Retinal vessel segmentation using neural network. IET Image Processing, 12(5) pp. 669678.

[45] Vaidya, Y. M., Swati, B. S. V. and Kantipudi, N. (2015): Moment Invariants based feature techniques for segmentation of retinal images using supervised method. International Conference on Industrial Instrumentation and Control (ICIC), pp. 1373-1377.

[46] Vincent ,V. C.; D. R.; M. S.S. \& A. J., (2019): Detection of Blood Vessels in Retinal Images using Line Tracking Algorithm, 2019 2nd International Conference on Intelligent Computing, Instrumentation and Control Technologies (ICICICT), pp. 967-974.

[47] Wang, Fengling. (2017): Comparative study on digital image enhancement for virtual restoration of mural painting." International Journal of Engineering and Technical Research, 7(12).

[48] World health organization (WHO) Report, 10 August (2021): [Online]. Available: https://www.who.int/

[49] Yang Y, Su Z, Sun L. (2010): Medical image enhancement algorithm based on wavelet transform. Electronics Letters 46,120-1.

[50] Zhou Y, Shi C, Lai B, Jimenez G. (2019): Contrast enhancement of medical images using a new version of the World Cup Optimization algorithm. Quant Imaging Med Surg , 9(9):1528-1547.

\section{Authors Profile}

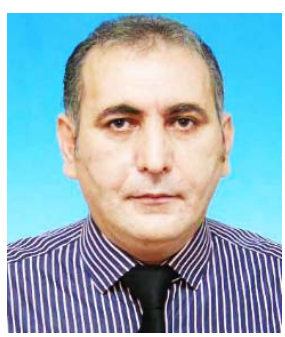

Dr. Nabhan D. Salih, academic at the Faculty of Computing and Informatics at Multimedia University. He received his Ph.D (2010) from Faculty of Engineering and Technology (Multimedia University) Field of study "Image Processing" and M.Sc. degree from University Putra Malaysia, Computer Science Field of study "Multimedia". His current research area is CBIR and Image Processing. He is interested in Video and Image Processing, Computer Vision and Multimedia Technology and Applications.

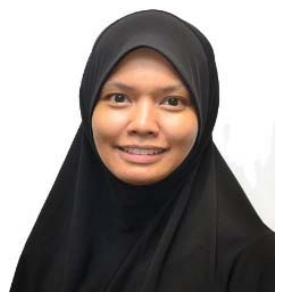

Dr. Wan Noorshahida Mohd Isa is currently a Senior Lecturer at the Faculty of Computing and Informatics, Multimedia University, Malaysia. She received her B.Sc. in Electrical Engineering from the Vanderbilt University, USA. the M.Sc. degree from University of Southampton in the Great Britain and Ph.D. degrees from Multimedia University, Malaysia in 2014. She has active research in the areas of data-driven machine learning techniques, computational intelligence, and pattern recognition systems and algorithms involving videos, images, and signal processing. 


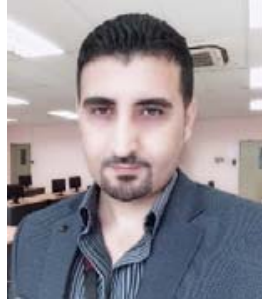

Dr. Marwan D. Saleh is an Assistant Professor at the University of Southampton Malaysia. Previously, he worked as a senior lecturer at Management and Science University (MSU) starting from 2019 until 2021. He also has extensive experience in computing industry. He worked as an IT Consultant for about 4 years. He received his B.Sc. degree in Computer Science in 2004 from Al-Mustansiriyah University, Iraq, and the M.Sc. and Ph.D. degrees in Information Technology from Multimedia University, Malaysia in 2010 and 2016 respectively. His principal research interests are in the fields of digital image analysis, pattern recognition, and computer vision. 